\title{
Chemometric differentiation of coffee varieties using gas sensors based on polymer-modified piezoelectric quartz crystals
}

\author{
Sharlene R. Cabanilla ${ }^{1}$ E Fortunato Sevilla III ${ }^{1,2}$ \\ ${ }^{1}$ Graduate School; ${ }^{2}$ Research Center for the Natural Sciences, University of Santo Tomas, España \\ Boulevard, 1015 Manila, Philippines
}

\begin{abstract}
Coffee is the most popular beverage worldwide, enjoyed primarily because of its aroma and flavor. Aroma is commonly used as the basis of coffee quality, and its evaluation is usually carried out by an expert sensory panel. Coffee aroma can also be assessed by means of an electronic nose. The electronic nose generates an electrical signal in the presence of the substances causing the aroma.

This study aimed to apply a self-assembled electronic nose system for the discrimination of the different varieties of coffee in the Philippines. Samples of the four varieties of coffee grown in the Philippines, i.e. Arabica, Robusta, Excelsa, and Liberica, were obtained from several regions in the country. The electronic nose system that was employed was based on six piezoelectric quartz crystals coated (PQC) with sensing elements of different polarities [polyethylene glycol (PEG), polyvinyl chloride (PVC), polymethylacrylate (PMAA), Fluorosil (PVC:Fluor), Silica (PVC:Silica) and Cat-Ex (PVC:CatEx)]. The following parameters involved in the measurement were optimized: weight of sample, flow rate of carrier gas, sample holder, fineness of sample, thickness of sensing elements and incubation time. Distinct radar plot quality profiles were obtained for the different varieties. Chemometric analysis of the responses of the electronic nose were carried out using principal component analysis (PCA) and dendogram cluster analysis. The developed group of sensors was able to categorize the different coffee varieties.
\end{abstract}

Keywords: coffee variety, variety discrimination, piezoelectric quartz crystals, gas sensors

\section{INTRODUCTION}

Coffee is the most widely consumed beverage in the world, with about 2.25 billion cups being drunk daily around the world [1]. Its popularity is attributed to its known physiological effects, as well as to its pleasant taste and unique aroma

*To whom correspondence should be addressed: cbdecena@ust.edu.ph / emyambao@ust.edu.ph
[2]. The unique aroma is warm and inviting, and sometimes described as the reminiscent of fruits, flowers, chocolates and even caramels. It is produced by a number of complex pyrolytic reactions during the roasting process.

There are 60 coffee plant varieties that can be found in the world; however, only four of these varieties are prominent in the global coffee beans 
trading. These are the Arabica, Excelsa, Liberica, and Robusta, all of which are grown in the Philippines. Each of these varieties has its distinct aroma. The volatiles that make up the aroma of these coffees play can be considered a "fingerprint" of the product.

Coffee contains more than a thousand volatile compounds that belong to different chemical families, including acids, alcohols, aldehydes, esters, furans, ketones, pyrroles, pyridines, phenolic, sulfur compounds, and etc. [3, 4]. However, only a small number of these compounds are responsible for the coffee aroma. That is why, even though considerable efforts have been applied to chemically analyze the compounds related to coffee aroma, still its identification and discrimination remains extremely difficult especially when using classical methods of analysis.

Coffee aroma has been traditionally assessed through sensory evaluation by trained human panels. This method is subjective, timeconsuming, expensive and with low reproducibility [5]. Gas chromatography coupled with mass spectrometry (GC-MS) has been widely used for the characterization of coffee aroma. However, the cost and complexity of the instrumentation present the main drawbacks for this method [6].

A technology has emerged that has been recognized to be useful for coffee characterization. This involves the electronic nose (EN) which is made up of an array of sensors that produce an electronic response in the presence of gases or vapors [6, 7]. A variety of sensors have been employed, but the most common ones are based on metal oxide semiconductors, conducting polymer and piezoelectric quartz crystals [8]. The responses of these sensors generate a pattern which provides information about the material being analyzed.
The electronic nose has found several applications to coffee analysis. It was used to discriminate commercial coffee blends and the roasting levels of coffee [8], to classify Arabica coffee from different countries and their blends [6], to evaluate the ripening of blends of moncultivar Arabica coffee [9], to differentiate several brands of Expresso coffee [10], to distinguish varieties of Arabica and Robusta coffee [11, 12], to identify different brands of Robusta, Brazilian and Colombian coffee [13] and to detect samples of Colombia coffee with "cup defects" [5]. The electronic noses employed in these applications were based on metal oxide semiconductor films.

In this paper, an array of polymer-modified piezoelectric quartz crystal sensors is employed for the discrimination of the four varieties of Philippine. This sensor system can be useful in the authentication of the coffee varieties employed in the preparation of coffee blends. It can also provide a basis for characterizing the quality of the coffee blends.

\section{EXPERIMENTAL METHOD}

Materials. All chemicals used in this study were analytical grade reagents: acetone (Merck), tetrahydrofuran, chloroform and toluene (Sigma Chemicals Co.). These chemicals were used as received and no further purification was done. The following polymers were used as coating for the quartz crystal sensor: polyvinyl chloride (BDH Limited Poole), polyethylene glycol PEG6000 (Ajax Chemicals), Florisil (SEP-PAK cartridge, Waters), cation exchange resin (LCSCX cartridge, Supelco), silica, (SEP-PAK cartridge, Waters) and polymethyl methacrylate. High purity nitrogen gas (Consolidated Industrial Gases, Inc.) was used as a carrier gas in the measurement process. Distilled water was used for washing bare crystals.

The quartz crystals (Beijing Chenjing Electronics Co., Ltd) were AT-cut, had polished gold 
electrodes on both sides (4 mm diameter and $1 \mu \mathrm{m}$ thickness), and had a resonance frequency of about $9 \mathrm{MHz}$.

The coffee samples consisted of different brands of commercial monovarietal coffee beans which were purchased in different provinces of the Philippines where each variety is known to grow - Arabica (A) from Baguio City; Liberica (L) from Batangas City; Excelsa (E) and Robusta (R) from Cavite City. The coffee beans were ground using a grinder (KRUPS F203), and the ground coffee was passed through standard test sieves (AST E-11 Specification) to obtain samples with different particle sizes - $841 \mu \mathrm{m}$, $595 \mu$, and $425 \mu \mathrm{m}$ from number 20, 30, and 40 sieves, respectively.

Sensor preparation. The sensor consisted of a piezoelectric quartz crystal coated on its two sides with a thin film of a polymer. The quartz crystal was first washed with distilled water, and then with acetone. The cleaned crystal was dried through a stream of nitrogen gas. The two sides of the crystal were coated with the sensing polymer using the spin coating method. The coated crystal was air-dried for a few minutes, and then a gentle stream of nitrogen gas was passed through it to completely dry the crystal.

The sensing material was prepared by dissolving the polymer in a suitable solvent in a certain ratio. Polyethylene glycol and polyvinyl chloride (PVC) were dissolved in chloroform and tetrahydrofuran, respectively, at a ratio of 2:1 $(\mathrm{w} / \mathrm{v})$. The other polymers (silica, florisil, cationexchange resin and polymethyl methacrylate) were mixed with PVC as a binder and tetrahydrofuran as solvent at a ratio 6:2:1 (w/w/ $\mathrm{v})$. The solutions were homogenized by means of an ultrasonic bath.

Instrumentation. A schematic diagram of the instrumentation employed in this study is presented in Fig. 1. It included a sample cell, a sensor chamber, a Pierce oscillator circuit and a frequency counter interfaced to a computer system. Nitrogen gas was used as a carrier gas which flushed the headspace vapor above the ground coffee in the sample cell into the sensor chamber. The sensor chamber contained a mounted polymer-coated quartz crystal which was connected to the oscillator circuit. The oscillator circuit which was based on a CMOS IC enabled the oscillation of the quartz crystal, and the frequency counter (Agilent IntuiLink Counter 53181A) recorded the resonance frequency of the quartz crystal.

Measurement procedure. Nitrogen gas was initially passed directly through the sensor chamber to obtain the baseline reading of the sensor system. It then passed through the sample cell which contained the coffee sample to transport the headspace vapor to the sensor chamber. The sensor response was monitored until it reached a steady state. After each measurement, the sensor chamber was purged with nitrogen gas for $20 \mathrm{~min}$. The difference in the frequency readings in the presence and in the absence of the coffee sample cell constituted the sensor response to the coffee sample. This difference which is denoted as $\Delta \mathrm{F}$ is related to the change in the mass loading on the quartz crystal sensor due to its interaction with volatile organic compounds from the headspace of the

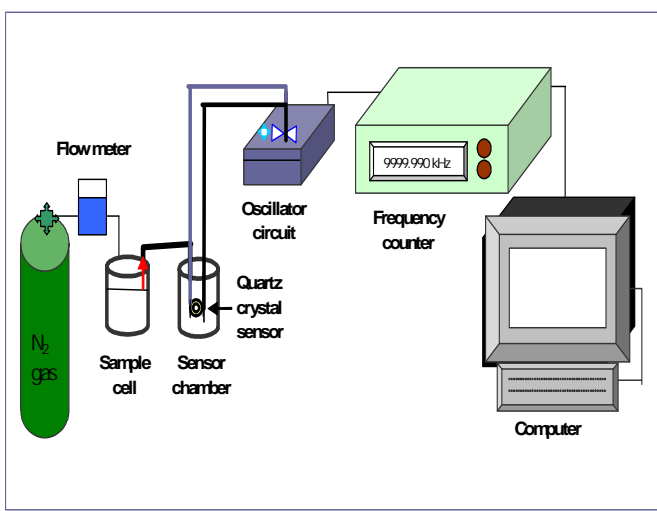

Figure 1. Schematic diagram of the instrumentation used in the measurements. 
coffee sample. The response value of the electric nose was recorded and analyzed using graphical methods and multivariate analysis.

\section{Results and discussion}

Sensor response. The sensors responded instantly to the headspace vapors of the coffee samples and attained a steady state value within a period of 70-100 sec. The sensors exhibited good reversibility and good reproducibility (rsd $=5-10 \%, n=3$ ). The response of the sensors was influenced by parameters of the sensor preparation, sample preparation and measurement procedure.

The sensor response was dependent on the amount of the polymer coating on the surface of the quartz crystal. The polymer functioned as the recognition element of the sensor. For all the polymeric reagents, with the exception of the cation-exchange/PVC, the optimum volume that must be applied on each side of the crystal was found to be $5 \mu \mathrm{L}$. For the cation-exchange PVC reagent, the optimum volume was $15 \mu \mathrm{L}$. Larger volumes caused lower responses due to the resultant thick coating which restricted the diffusion of the analyte into the sensing layer.

The amount and fineness of the coffee sample also affected the sensor response. The response varied with the amount of the sample used, exhibiting a maximum response from a $1.00 \mathrm{~g}$ sample. Greater amounts of sample produced a lower response since the sensing chamber had a constant volume and the volume of the headspace was decreased as the amount of the sample was increased. The sensor response increased as the fineness of the sample was increased, the greatest response being observed from the sample with particles having a diameter of $425 \mu \mathrm{m}$ or less. The sample with finer particles possessed a greater surface area which enabled the released of more volatile components into the headspace.
The incubation time of the coffee in the sample vessel was found to have an effect on the sensor response. The sensor response increased, as the incubation period was lengthened. During incubation, the coffee volatiles vaporize and equilibrate with the solid sample. A maximum response was observed for an incubation time of $5 \mathrm{~h}$. Extending the incubation period beyond $5 \mathrm{~h}$ did not cause a change in the sensor response, indicating equilibrium of the vapor with the solid phase was achieved within $5 \mathrm{~h}$. For practical reasons, the sample was incubated in the vessel overnight.

The flow rate of the nitrogen carrier gas was found to influence the sensor response. The highest response was observed for a flow rate of $0.5 \mathrm{~mL} \mathrm{~min}^{-1}$. At higher flow rates, the sensor response decreased which can be associated with a decreased concentration of the coffee volatiles in the gas stream. This behavior can be attributed to the dilution effect of the greater amount of carrier gas flowing within a given time.

Variation with coffee variety. The response of the different sensors varied with the variety of the coffee samples (Fig. 2). This behavior indicates that the headspace composition of each variety is different. This is consistent with the distinct aroma exhibited by each coffee variety. The response obtained from Arabica

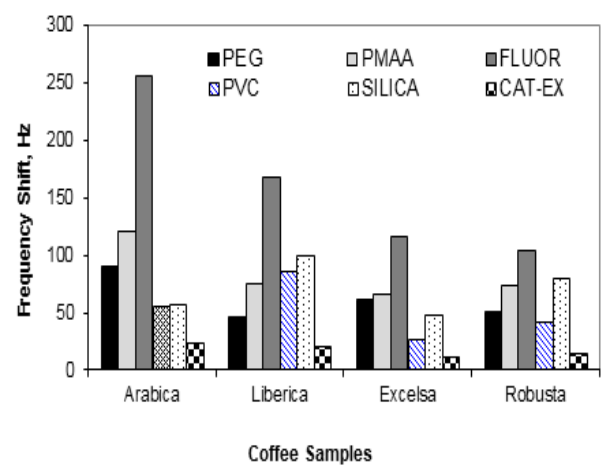

Figure 2. Response of the different sensors to each coffee variety 
coffee was the highest, which can be due to the presence of more volatile organic compounds in the headspace.

Among the six polymer coatings that had been utilized, PVC/Fluor gave the highest response to all the coffee varieties while PVC/CatEx gave the lowest response. PVC/CatEx features an ionic moiety which interacts selectively with charged species, which rarely occur in the vapor phase at room conditions.

A difference in the pattern of the bar graphs of the sensor responses to the different coffee varieties can be discerned and can be used to discriminate the different varieties. However, visual discrimination of the different patterns cannot be carried out easily. Another technique for pattern recognition involves the presentation of the data in a radar plot. Distinct shapes, which are easily recognizable, were exhibited by the different coffee varieties, and be used as a chemical signature or fingerprint for the headspace of each variety.

A chemometric approach provides a quantitative means for the differentiation of the responses of the chemoresistive sensors towards the different wood samples. Principal component analysis (PCA) was the pattern recognition technique adopted in this study. It is a widely used pattern recognition technique for analyzing, classifying and reducing the dimensionality of numerical data sets in a multivariate problem. Figure 4 shows the PCA score graph for the first two principal components derived from responses of the different sensors to the wood samples. The four different varieties formed well separated clusters which are easily recognized.
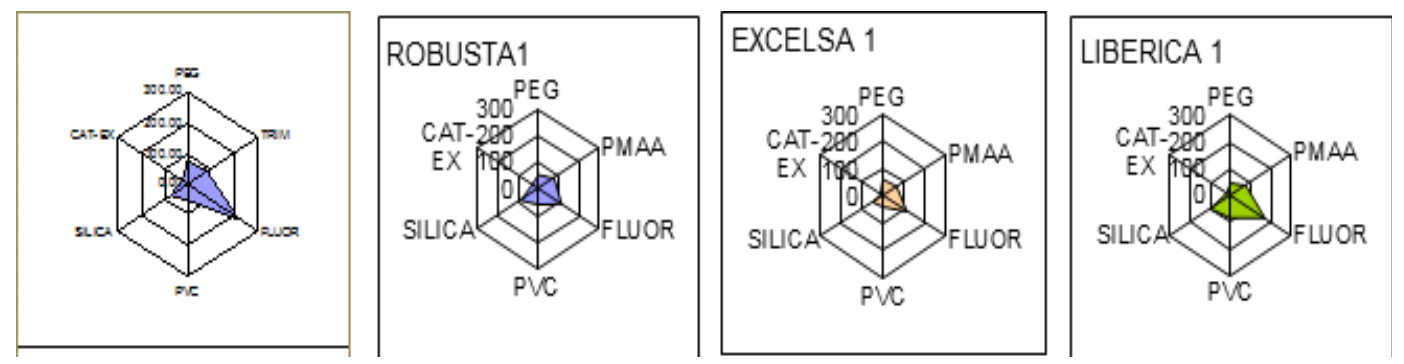

Figure 3. Radar plots of the response of the sensors to the different coffee varieties

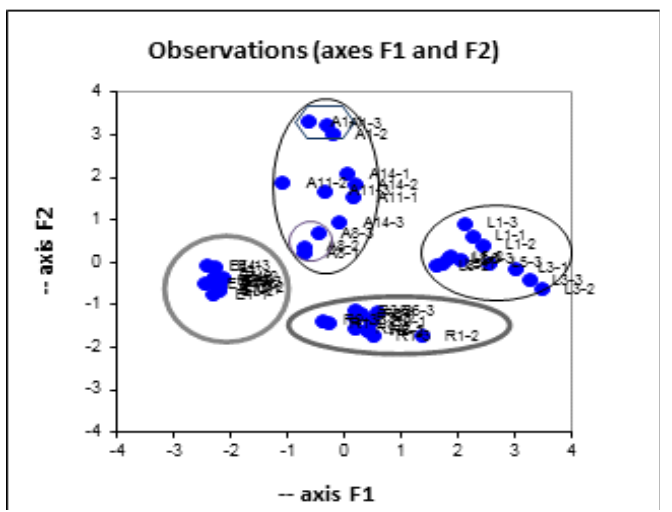

Figure 4. Score plot of the coffee samples: Arabica (A), Liberica (L), Excelsa (E), Robusta (R)

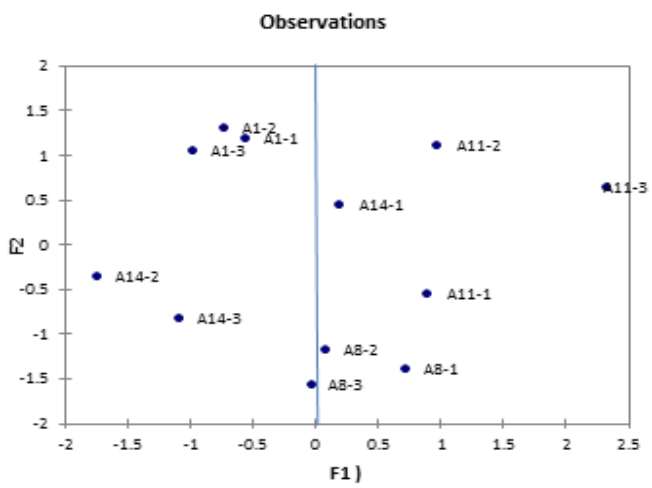

Figure 5. Score plot of the sensor responses for the Arabica samples. 
The first two principal components accounted for $76 \%$ of the variance. The principal component F1 differentiated very well the Excelsa and the Liberica varieties. The Excelsa coffee samples lie on the negative part of the F1, lie the axis, whereas the Liberica samples were in the positive part of the axis. The Arabica and Robusta varieties were distinguished well by the F2 principal component. In the positive part of F2 lie the Arabica coffee samples, while in the negative part of F2 is where the measurement gathered from the Robusta coffee samples lie. These distinct clusterings indicate the difference in the headspace composition of each variety.

A wide spread of the data points for the Arabica coffee samples is noticeable in the score plot. A closer scrutiny revealed clustering based on the roasting method. Principal component F2 differentiated dark roasted coffee (A8), from medium roasted coffee (A14), French roasted coffee (A11) and traditional roasted coffee (A1). A principal component analysis of the sensor responses for the Arabica samples yielded a score plot (Fig. 5) which highlighted the distinction based on the degree of roasting of the different coffee samples.

\section{CONCLUSION}

Coffee samples of different varieties were differentiated by a set of polymer-coated piezoelectric quartz crystals exposed to the headspace of the samples. Principal component analysis highlighted the discrimination of the sensor responses. The chemometric analysis also distinguished the coffee samples based on the degree of roasting. The differentiation is the different interaction of the volatile organic compounds present in the headspace of the coffee with the reagent phase.

\section{REFERENCES}

[1] Gunter MJ, Murphy N, Cross AJ, Dossus L, et al. Coffee Drinking and Mortality in 10 European Countries A Multinational Cohort Study. Annals of Internal Medicine 2017, 167:236-252.

[2] Silvarolla M, Mazzafera P, \& Frazuoli L. A naturally decaffeinated arabica coffee. Nature 2004, 429:826.

[3] Lopez-Galilea I, Fournier N, Cid C, \& Guichard E. Changes in headspace volatile concentrations of coffee brews caused by the roasting process and the brewing procedure. Journal of the Science of Food and Agriculture 2006, 54:85608566.

[4] Rocha S, Maeztu L, Barros A, Cid C, \& Coimbra MA. Screening and distinction of coffee brews based on headspace solid phase microextraction/ gas chromatography/principal component analysis. Journal of the Science of Food and Agriculture 2003, 84:43-51.

[5] Rodríguez J, Durán C, \& Reyes A. Electronic nose for quality control of Colombian coffee through the detection of defects in "Cup Tests". Sensors 2010, 10:36-46.

[6] Pardo M \& Sberveglieri G. Coffee Analysis With an Electronic Nose. IEEE Transactions on Instrumentation and Measurement 2002, 51:1334-1339.

[7] Gardner JW, Shurmer HV, \& Tan TT. Application of an electronic nose to the discrimination of coffees. Sensors and Actuators B 1992, 6:7175.

[8] Gardner J \& Bartlett P. Electronic Noses. (Oxford, UK: Oxford University Press, 1999).

[9] Falasconi M, Pardo M, Sberveglieri G, Riccò A, \& Bresciani A. The novel EOS 835 electronic nose and data analysis for evaluating coffee ripening. Sensors and Actuators B: Chemical 2005, 110:73-80.

[10] Pardo M, Niederjaufner G, Benussi G, Comini E, Faglia G, Sberveglieri G, Holmberg M, \& Lundstrom I. Data preprocessing enhances the classification of different brands of espresso coffee with an electronic nose. Sensors and Actuators B: Chemical 2000, 69:397-403.

[11] Aishima T. Aroma discrimination by pattern recognition analysis of responses from semiconductor gas sensor arrays. Journal of Agricultural and Food Chemistry 1991, 39:752756. 
[12] Buratti S, Sinelli N, Bertone E, Venturello A, Casiraghi E, \& Geobaldo F. Discrimination between washed Arabica, natural Arabica and Robusta coffees by using near infrared spectroscopy, electronic nose and electronic tongue analysis. Journal of the Science of Food and Agriculture 2014, 95:2192-200.
[13] Ulmer H, Mitrovics J, Noetzel G, Wiemar U, \& Gopel W. Odours and flavours identified with hybrid modular sensor systems. Sensors and Actuators B: Chemical 1997, 43:24-33. 